\title{
System-independent material classification through X-ray attenuation decomposition from spectral X-ray CT
}

Jumanazarov, Doniyor; Koo, Jakeoung; Busi, Matteo; Poulsen, Henning Friis; Olsen, Ulrik Lund; lovea, Mihai

Published in:

NDT and E International

Link to article, DOI:

10.1016/j.ndteint.2020.102336

Publication date:

2020

Document Version

Early version, also known as pre-print

Link back to DTU Orbit

Citation (APA):

Jumanazarov, D., Koo, J., Busi, M., Poulsen, H. F., Olsen, U. L., \& lovea, M. (2020). System-independent material classification through X-ray attenuation decomposition from spectral X-ray $\mathrm{CT}$. NDT and $E$ International, 116, [102336]. https://doi.org/10.1016/j.ndteint.2020.102336

\section{General rights}

Copyright and moral rights for the publications made accessible in the public portal are retained by the authors and/or other copyright owners and it is a condition of accessing publications that users recognise and abide by the legal requirements associated with these rights.

- Users may download and print one copy of any publication from the public portal for the purpose of private study or research.

- You may not further distribute the material or use it for any profit-making activity or commercial gain

- You may freely distribute the URL identifying the publication in the public portal 


\title{
System-independent material classification through X-ray attenuation decomposition from spectral X-ray CT
}

\author{
Doniyor Jumanazarov ${ }^{a, d}$, Jakeoung $\mathrm{Koo}^{b}$, Matteo Busi ${ }^{c, a}$, Henning F. Poulsen ${ }^{a}$, Ulrik L. Olsen ${ }^{a}$ and \\ Mihai IOVEA $^{d, *}$ \\ ${ }^{a}$ Technical University of Denmark, DTU Physics, 2800 Kgs. Lyngby, Denmark \\ ${ }^{b}$ Technical University of Denmark, DTU Compute, 2800 Kgs. Lyngby, Denmark \\ ${ }^{c}$ Paul Scherrer Institute, Forschungsstrasse 111, 5232 Villigen, Switzerland \\ ${ }^{d}$ ACCENT PRO 2000 s.r.l. (AP2K), Nerva Traian 1, K6, Ap. 26, Bucharest, S3, ROMANIA 031041
}

\section{ARTICLE INFO}

\section{Keywords:}

Spectral X-ray CT

Electron density

Effective atomic number

Photon counting detectors

Security screening

\begin{abstract}
A B S TRACT
We present a method for material classifications in spectral X-ray Computed Tomography (SCT) taking advantage of energy-resolving 2D detectors to simultaneously extract the energy dependence of a material's linear attenuation coefficient (LAC). The method employs an attenuation decomposition presented by Alvarez et al., and estimates system-independent material properties of electron density $\left(\rho_{\mathrm{e}}\right)$ and effective atomic number $\left(Z_{\text {eff }}\right)$, independent of the scanner, from the energy-dependent LAC measurements. The method uses a spectral correction algorithm and the energy range is truncated to exclude bins with photon starvation and spectral distortion present even after correction of detector response. A novel technique of energy bin selection is used for optimized classification performance. The method is tested against another SCT classification method called SRZE for inspecting materials in the range of $6 \leq Z_{\text {eff }} \leq 23$. Our method aims at an increase in the speed of pot processing workflow after the data acquisition, and it achieves explicitly up to 32 times better time efficiency for the reconstruction with comparable accuracy for a range of materials important in threat detection.
\end{abstract}

\section{Introduction}

The discrimination between innocuous liquids and precursors for homemade explosives is a difficult challenge for civil aviation safety screenings, since both types of materials are organic liquids with similar $\mathrm{x}$-ray attenuation. The detection of these threats and other traditional explosives requires a technology that can provide accurate material features [1]. Over the years, X-ray Computed Tomography (CT) has been widely used for Non-Destructive Testing (NDT), as well as in the field of illegal material detection [2]. Conventional CT measures the effective linear attenuation coefficient (LAC) across a broad energy range since the typical X-ray sources generate a polychromatic beam. The Hounsfield unit is often used with conventional CT. It represents the attenuation spectra with a single value, with an uncertainty that is larger than the inter-material variation for some materials [1]. In 1976, Alvarez et al. [3] showed a method for CT that decomposes LAC into photoelectric absorption and Compton scattering basis functions and they demonstrated that an alternative material characterization with just two parameters density, $\rho$, and effective atom number, $Z_{\text {eff }}$ can define the whole spectral range of the LAC. Dual-energy CT (DECT) measures two different signals in the attenuation spectrum and can be used to reconstruct both parameters [4-14]. The Dual-Energy detector as shown in Fig. 1 have overlapping low- and high-energy spectrums and the Dual-Energy Systems suffer from poor energy separation.

\footnotetext{
*Corresponding author.

@doniyor.jumanazarov@fysik.dtu.dk (D. Jumanazarov); jakoo@dtu.dk (J. Koo); matteo.busi@psi.ch (M. Busi); hfpo@fysik.dtu.dk (H.F. Poulsen); ullu@dtu.dk (U.L. Olsen); office@accent.ro (M. IOVEA) ORCID(s): 0000-0003-1583-7375 (M. IOVEA)
}

The material classification through the effective LAC with dual-energy sandwich detectors, detectors currently in use in security screening systems is a system-dependent solution $[4,15]$ since it depends on source spectrum, filtration, and detector's efficiency. Recently, Azevedo et al. [4] proposed a method that estimates $\left(\rho_{\mathrm{e}}, Z_{\text {eff }}\right)$ from dual-energy CT known as the System-Independent $\rho_{\mathrm{e}} / Z_{\text {eff }}$ (SIRZ), independent of the scanner, presented in Fig. 1 and Champley et al. [16] have developed the method further (SIRZ-2). Like all DECT this method requires two successive scans with different source kilovoltage peaks and filtration, and additionally the calibration of the detector's spectral response by using a group of reference materials. In the range of $Z_{\text {eff }}$ between 6 and 20, and energies up to $200 \mathrm{keV}$, the accuracy error and precision errors for estimating both $\left(\rho_{\mathrm{e}}, Z_{\text {eff }}\right)$ features in SIRZ were less than 3\% and 2\% respectively, while the SIRZ-2 accuracy errors were $0.7 \%$ for $\rho_{\mathrm{e}}$ and $<1.5 \%$ for $Z_{\text {eff }}$, and the SIRZ-2 precision errors for both were up to $2.3 \%$.

The development of cadmium telluride (CdTe) energydiscriminating imaging detectors has paved the way for Spectral CT [17-19]. Spectral CT with an energy resolved photon counting detector (PCD) is proved to have a number of advantages over dual-energy CT for improving material identification [20,21]. This has resulted in a large interest in PCDs within security applications [22, 23]. In material identification by radiography, Rinkel et al. [24] presented through experimental data that the false detection rate when using 90 energy bins with a PCD was reduced by a factor of up to 2 and more than 3 (for thin objects) compared to an optimised dual-energy bins approach applied both on the same PCD data and to a dual-layer sandwich detector, re- 
spectively. Beldjoudi et al. [20, 25] showed through simulations in Radiography that PCDs with data condensed into two energy bins enhanced recognition of single materials by over 50\% relative to dual-layer sandwich detectors. Increasing the number of energy bins resulted in a further rise of $80 \%$ in material identification with 90 energy bins compared to dual-layer sandwich detectors. They found that the poor spectral separation in the dual-layer sandwich detectors appears to be the main reason for the inferior performance. In contrast PCDs exhibit a significantly better spectral resolution. As expected, using energy bins with a width below the energy resolution of the detector did not lead to great improvement in identification performances. However, using a higher number of energy bins or narrow bin widths provided a more robust configuration, regardless of the thickness and nature of the material. A simulation study by Wang et al. [26] showed how to select the energy bin widths to optimize the performance of basis material decomposition in material recognition. To check the accuracy and precision of the method in estimating the thickness of the two basis materials, they investigated five different energy bin combinations to define energy bins setting energy bin thresholds for each; bins of equal width, bins of equal incident photon counts, bins of equal incident photon intensity, bins of equal photon count and bins with equal photon intensity after passing through $3 \mathrm{~cm}$ of PMMA. They found the best results when using energy bins with similar incident photon counts. Martin et al. [23] introduced a learning and adaptive model-based method to optimally select the bins for material characterization from multi-energy data.

The MultiX ME100 (MultiX, Neuillysur-Seine, France) PCD is a good candidate for high flux measurements to extract a spectrally resolved LAC. Brambilla et al. [27] implemented a basis material decomposition method for material characterization using this detector. This method requires a calibration of the detector's spectral response for a group of reference materials, and only estimates $Z_{\text {eff }}$ accurately in 3D measurements. Based on the Multix detector the SIRZ method was further extended into the Spectral $\rho_{\mathrm{e}} / Z_{\text {eff }}$ Estimation (SRZE) method presented by Busi et al. [28], which estimates system-independent $\left(\rho_{\mathrm{e}}, Z_{\text {eff }}\right)$ directly from the spectral LACs in Spectral CT (in Fig. 1). The accuracy of the SRZE method relies on the accurate measurement of spectral LACs, and it decreased for materials with smaller $Z_{\text {eff }}$ due to detector response artefacts at lower energies, where the contrast of low $Z_{\text {eff }}$ material is also highest [28].

The method presented in this work aims to estimate the system-independent material features $\left(\rho_{\mathrm{e}} Z_{\text {eff }}\right)$, independent of the system or specifics of the scanner, such as the x-ray spectrum. It uses the attenuation decomposition presented by Alvarez et al. [3] for Spectral CT. Fig. 1 illustrates the principles of the novel method, named system-independent material classification through attenuation decomposition (SIMCAD). As illustrated, this approach is based on nonoverlapping optimized bi-energy bins separated by a gap. Wang et al. $[29,30]$ presented that for PCDs that discriminate between low and high energy photons, having a gap between energy thresholds is useful for material separability. Photons with energies that lie in this gap should either be discarded or counted separately to enhance performance. Therefore, the energy range falling into this gap was discarded in the optimized bi-energy bins approach in our work. The method requires a calibration step to find the energydependent basis functions of the decomposed attenuation by using a set of reference materials. Materials are then classified through the two parameters ( $\rho_{\mathrm{e}}$ and $Z_{\text {eff }}$ ) that can fully describe the wide range of materials that may appear in baggage scanners [31].

In this work, we compare the performance of the SRZE and SIMCAD methods over a common set of materials using experimental data. The SRZE method reaches the optimal performance at 64 energy bins and provides noticeably lower classification performance with two energy bins [32]. The SIMCAD method utilizes optimized bi-energy bins for comparable classification performance, and uses at least 32x shorter time for the tomographic reconstruction after the data acquisition directly due to a lower number of energy bins. The speed makes the method compatible with the requirements for applications where rapid scanning is required. Check-in baggage inspection at airports is such an application where the time and computational resources required for spectral reconstruction represents a major issue [33].

\section{Theory and Methods}

The aim of this section is firstly to define the systemindependent material features used to classify materials from experimental data, and secondly to present instrumentation and methods with the detection algorithm.

\subsection{Photoelectric-Compton decomposition}

Bragg and Peirce (1914) [34], Owen (1919) [35] and Richtmyer and Warburton (1923) [36] established the original concept of the dependence of photon interactions upon atomic number (Z), and this concept has been implemented in many radiation studies [37]. For a computerized tomography system, Alvarez et al. [3] showed empirically over the range of 30-200 keV that the LAC could be decomposed in the form:

$$
\mu(E)=a_{1} \frac{1}{E^{3}}+a_{2} f_{\mathrm{KN}}(E),
$$

where $f_{\mathrm{KN}}(E)$ is the Klein-Nishina function

$$
\begin{aligned}
f_{\mathrm{KN}}(\varepsilon)=\frac{1+\varepsilon}{\varepsilon^{2}}(2 & \left.\frac{1+\varepsilon}{1+2 \varepsilon}-\frac{\ln (1+2 \varepsilon)}{\varepsilon}\right) \\
& +\frac{\ln (1+2 \varepsilon)}{2 \varepsilon}-\frac{1+3 \varepsilon}{(1+2 \varepsilon)^{2}}
\end{aligned}
$$

and $\varepsilon=E / 511 \mathrm{keV}\left(\varepsilon=E / m_{\mathrm{e}} c^{2}\right)$ is the reduced energy of the incident photon. The functions $1 / E^{3}$ and $f_{\mathrm{KN}}(E)$ approximate the energy dependence of the photoelectric absorption and Compton scattering, respectively. Excluding 



Figure 1: Illustration of the dual-energy CT, SRZE and SIMCAD methods. Left: in dual-energy X-ray CT, the sample attenuation is measured at low- and high energies with two different spectra that are overlapping and thus reduces the estimation performance $[24,25]$. Center: in classical spectral X-ray CT, the sample attenuation is measured at multiple energy bins using PCDs [23, 28]. Right: in the optimized bi-energy bins approach to spectral X-ray CT, the sample attenuation is measured without overlap at lowand high energies with PCDs in a single acquisition. The selection of the upper and lower thresholds of low- and high-energies, respectively $b_{1}$ and $b_{2}$ is discussed in the subsection 3.2 .

the effect of absorption edges, they further showed that the photoelectric coefficient, $a_{1}$ and the Compton scattering coefficient, $a_{2}$ could be expressed in terms of physical parameters as:

$$
a_{1} \approx \mathrm{K}_{1} \frac{\rho}{A} Z^{n}, a_{2} \approx \mathrm{K}_{2} \frac{\rho}{A} Z
$$

where $\mathrm{K}_{1}$ and $\mathrm{K}_{2}$ are constants, $\rho$ is mass density $\left(\mathrm{g} / \mathrm{cm}^{3}\right)$, $A$ is atomic mass and $Z$ is atomic number. $n$ is the exponent for photoelectric attenuation (per atom). We optimize $n$ for each of the classification approaches depending on how many energy bins are used. $a_{1}$ and $a_{2}$ represent only approximate expressions. The insertion of $a_{1}$ and $a_{2}$ from Eq. (3) into Eq. (1) yields the LAC:

$$
\mu(E)=\frac{Z}{A} \rho\left(Z^{n-1} p(E)+c(E)\right),
$$

where

$$
p(E)=\mathrm{K}_{1} \frac{1}{E^{3}}, c(E)=\mathrm{K}_{2} f_{\mathrm{KN}}(E) .
$$

$p(E)$ and $c(E)$ are photoelectric absorption and Compton scattering basis functions, respectively. The basis functions are empirical defined through experimental fit to data. In our calculations, we optimize the basis functions and parameter $n$ for the best classification performance and the resulting optimized values for all the multiple energy bins approaches are presented in the subsection 2.5 .

\subsection{Electron Density and Effective Atomic Number}

X-ray attenuation is proportional to a material's electron density [38]. The electron density is the number of electrons per unit volume (electron - mole $/ \mathrm{cm}^{3}$ ) and related to mass density, atomic number and atomic mass of an element as:

$$
\rho_{\mathrm{e}}=\frac{Z}{A(Z)} \rho,
$$

For a compound or mixture that has the composition of $N$ total different elements $i$ each with a number of atoms, $\alpha_{i}$, the electron density can be derived as

$$
\rho_{\mathrm{e}}=\frac{\sum_{i=1}^{N} \alpha_{i} Z_{i}}{\sum_{i=1}^{N} \alpha_{i} A_{i}} \rho .
$$

For compounds, the atomic number is referred to as effective atomic number, $Z_{\text {eff }}$ and a now classical parameterization was proposed by Mayneord (1937) [39] and Spiers (1946) [40] as

$$
Z_{\text {eff }}=\sqrt[l]{\sum_{i=1}^{N} r_{i} Z_{i}^{l}},
$$

where $N$ is the number of elements in the material, $Z_{i}$ is the atomic number for each element, $i, r_{i}$ is "relative electron fraction" contribution. The relative electron fraction of an element, $i$ is expressed as

$$
r_{i}=\frac{\alpha_{i} Z_{i}}{\sum_{j=1}^{N} \alpha_{j} Z_{j}},
$$

where $\alpha_{i}$ is the number of atoms that have atomic number $Z_{i}$.

No single formula of $Z_{\text {eff }}$ can characterize the alteration of photoelectric absorption cross section over the particular range of the photon energies and materials with the acceptable accuracy of interest in CT [37, 40-47]. The Compton interaction takes place with relatively unbound electrons for the photon energy range and materials relevant to CT Systems, and consequently the Compton scattering cross section is independent of chemical makeup of materials and only is a function of photon energy [41], as discussed in the subsections 2.1 and 2.5. Therefore, the exponent $l$ is optimized depending on the material, source spectrum and system features. Usually, this parameter, $l$ changes between 
2.94 and 3.8 based on experimental fits for different CT systems. In our calculations, $l$ is set to the value of the original Lehnmann parameterization, which is 3.8 [44]. Even though Eq. (8) neglects the coherent scatter part, this definition of $Z_{\text {eff }}$ based on the Lehnmann parameterization operates quite well because the total cross section is mostly governed by the Compton cross section for the set of our materials and photon energy range [44, 47]. Thus, we use two separate sets of parameters, $n$ and $l$ to define the photoelectric absorption cross section term, being optimized depending on the approach of multiple energy bins and single value for all the materials, respectively. In the subsection 3.1 , the dependence of the classification performance on exponent $l$ is presented and it is discussed how this exponent is selected in this work.

Substituting Eq. (6) into Eq. (4) we obtain a parameterized LAC for a compound material as

$$
\mu(E)=\rho_{\mathrm{e}}\left(Z_{\mathrm{eff}}^{n-1} p(E)+c(E)\right) .
$$

\subsection{Experimental setup and data correction.}

Fig. 2 illustrates the instrumentation. The experiments were performed in the 3D Imaging Center at DTU, Denmark. A Hamamatsu X-ray source was used operating at the acceleration voltage and the filament current set to $160 \mathrm{kV}$ and $0.5 \mathrm{~mA}$, respectively. The focal spot for these parameters is $75 \mu \mathrm{m}$. An aluminum filter of $2 \mathrm{~mm}$ thickness was used to remove photons with energies lower than the detector energy range. The beam was collimated into a fan beam geometry. The samples were placed on the rotation stage and scanned during the acquisition over a range of 360 degrees in discrete steps of $1^{\circ}$ increments. The source to detector distance (SDD) was set to $701 \mathrm{~mm}$ while the source to sample distance (SOD) was $500 \mathrm{~mm}$.

The detector system comprises five MultiX ME100 modules, each consisting of $1 \times 128$ pixels of size $0.8 \times 0.8 \mathrm{~mm}^{2}$. The number of energy bins is flexible and could be configured for the purpose of the experiment between 2 and 128 . In this work it was set to 128 with a width of $1.1 \mathrm{keV}$ each, evenly distributed in the energy range between 20 and 160 $\mathrm{keV}$. The energy resolution of the detector, defined by the Full Width at Half Maximum (FWHM) is $6.5 \%(8 \mathrm{keV}$ at $122 \mathrm{keV}$ ) by high X-ray fluxes [19]. In this work, the total integration time per projection is 8 seconds.

Charge sharing, weighting potential, escape peaks, pulse pile-up and incomplete charge collection in PCDs lead to data distortion [48]. These effects are related to energy and flux, and therefore, the data distortion affects the material's attenuation property. To correct for these spectral effects we used the correction algorithm presented by Dreier et al. [48]. Fig. 3 shows the raw and corrected source spectrum. The average counts integrated for the spectra was approximately $1.6 \times 10^{6}$ counts/pixel per second.

Table 1 lists the materials studied in this work as well as their reference $\rho_{\mathrm{e}}$ and $Z_{\text {eff }}$ values that were calculated by using Eq. (7) and Eq. (8), respectively. The material features presented in the table will be used as the reference values in the following sections. The distribution of $Z_{\text {eff }}$ for materials commonly found in a passen- ger bag peaks between 7-8. Most organic materials belong to this range [1]. The plastics used for the experiment were polymethyl methacrylate (PMMA), polytetrafluoroethylene (PTFE), polyvinylidene fluoride (PVDF), polycarbonate (PC), polyoxymethylene-C (POM-C), polyethylene terephthalate (PET) and polyoxymethylene-H (POM$\mathrm{H})$. These plastics were chosen since several explosives have similar chemical compositions, e.g. POM has been considered an explosive simulant [24]. The classification of plastics is thus an efficient gauge for a system's ability to identify explosive materials in luggage. The materials used in the work represents the same $Z_{\text {eff }}$ variation as various organic tissues [49] and none have K-edge absorption within the detector's energy range. Sample dimension are found in Table 1 through width $\times$ length or diameter for rectangular and circular samples respectively.

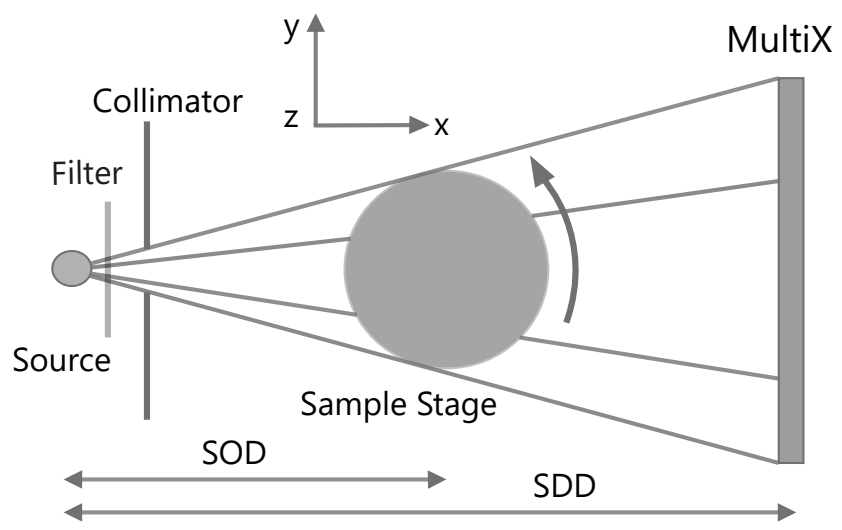

Figure 2: The illustration of the instrumental setup.

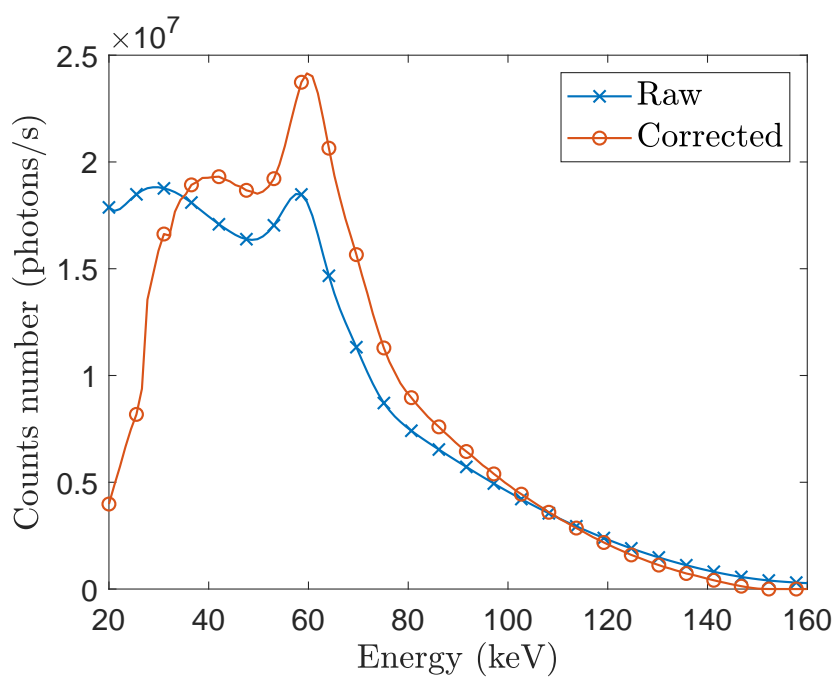

Figure 3: X-ray source spectra before and after applying spectral correction algorithm. The number of counts per second summed over all detector pixels is shown, measured through the MultiX ME100 PCD. 
Table 1

The list of all the materials scanned and processed through the method, and their physical properties $\rho_{\mathrm{e}}$ and $Z_{\text {eff }}$. The mass density, $\rho$ for the plastic materials was measured with uncertainties of $\pm 0.15 \%$. The mass densities for the rest of materials represent the theoretical values found in PubChem data, [50].

\begin{tabular}{llllll}
\hline Material & $\begin{array}{l}\text { Chemical } \\
\text { Formula }\end{array}$ & $\begin{array}{l}\text { Widthxlength/ } \\
\text { Diameter }(\mathrm{mm})\end{array}$ & $\rho\left(\mathrm{g} / \mathrm{cm}^{3}\right)$ & $\rho_{\mathrm{e}}\left(\mathrm{e}^{-} \mathrm{mol} / \mathrm{cm}^{3}\right)$ & $Z_{\text {eff }}$ \\
\hline PMMA & $\left(\mathrm{C}_{5} \mathrm{O}_{2} \mathrm{H}_{8}\right)_{n}$ & $40 \times 42$ & 1.18 & 0.636 & 6.60 \\
PTFE & $\left(\mathrm{C}_{2} \mathrm{~F}_{4}\right)_{n}$ & $9 \times 53.3$ & 2.16 & 1.039 & 8.50 \\
PVDF & $\left(\mathrm{C}_{2} \mathrm{H}_{2} \mathrm{~F}_{2}\right)_{n}$ & $9 \times 53.5$ & 1.8 & 0.9 & 8.01 \\
PC & $\left(\mathrm{CO}_{3} \mathrm{C}_{13} \mathrm{H}_{8}\right)_{n}$ & $8.2 \times 53.5$ & 1.19 & 0.615 & 6.48 \\
POM-C & $\left(\mathrm{CH}_{2} \mathrm{O}\right)_{n}$ & $9 \times 53.5$ & 1.42 & 0.758 & 7.07 \\
PET & $\left(\mathrm{C}_{10} \mathrm{H}_{8} \mathrm{O}_{4}\right)_{n}$ & $9 \times 53.5$ & 1.39 & 0.726 & 6.74 \\
POM-H & $\left(\mathrm{CH}_{2} \mathrm{O}\right)_{n}$ & $15.5 \times 53.3$ & 1.44 & 0.766 & 7.07 \\
Acetone & $\mathrm{C}_{3} \mathrm{H}_{6} \mathrm{O}$ & 20 & 0.785 & 0.432 & 6.44 \\
Hyd. Peroxide & $\mathrm{H}_{2} \mathrm{O}_{2}(50 \%)$ & 20 & 1.22 & 0.661 & 7.65 \\
Methanol & $\mathrm{CH}_{3} \mathrm{OH}$ & 20 & 0.792 & 0.446 & 6.86 \\
Water & $\mathrm{H}_{2} \mathrm{O}$ & 20 & 0.997 & 0.554 & 7.54 \\
Nitromethane & $\mathrm{CH}_{3} \mathrm{NO}_{2}$ & 20 & 1.14 & 0.597 & 7.27 \\
Aluminum & $\mathrm{Al}$ & 25 & 2.70 & 1.3 & 13 \\
Silicon & $\mathrm{Si}$ & 25 & 2.33 & 1.161 & 14 \\
Magnesium & $\mathrm{Mg}$ & 12.7 & 1.74 & 0.858 & 12 \\
Titanium & $\mathrm{Ti}$ & 12.7 & 4.51 & 2.071 & 22 \\
\hline
\end{tabular}

\subsection{Setting low- and high-energy thresholds and LAC extraction}

For each projection the photon counts $I_{k}$ of energy bin $k$ is converted to line integrals $L_{k}$ based on Lambert-Beer's law as:

$$
L_{k}(\vec{x})=-\log \frac{I_{k}(\vec{x})}{I_{0, k}(\vec{x})}, \quad k=1,2,3, \ldots, 128 ;
$$

where $I_{0, k}$ is the flat-field photon flux for energy bin $k$, i.e. the projection measured by the detector without inserting the sample. This is also referred as the source spectrum or the system's spectral response. $\vec{x}$ represents the 1D detector pixel array, the detector has total 128 energy bins.

The intensity in the gap spaces between two adjacent modules, with the width approximately equal to two pixels, were filled by neighbor interpolation. Slice reconstructions were performed individually for each energy bin from each line integral (or sinogram) by using the SIRT implementation in the ASTRA Toolbox [51] to extract LACs of materials. Fig. 4 presents how the LAC values are extracted from the histogram based on Gaussian curve fits after sample reconstruction. MATLAB ${ }^{\circledR}$ 's fitdist function with normal distribution was used to extract the LACs for all materials and for all approaches of the number of used energy bins. The ring artifacts in the reconstruction may be due to the difference in detector response for the pixels at crystal edges in the detector since the correction algorithm is optimized for the central pixels.

Fig. 5 shows measured and reference spectral LAC of PTFE plastic and aluminum with and without spectral correction. Additional spectral distortions appear at low- and high-energies, which cannot be totally corrected by the spectral correction algorithm. LAC deviations from the reference values at low- and high-energies could be due to detector flux variation and photon starvation, i.e. complete attenuation of photons [48]. Energy bins with those deviations are excluded by setting low- and high-energy thresholds, $E_{\mathrm{i}}$ and $E_{\mathrm{f}}$ that are kept constant for all materials. We note that there is a slight deviation between the measured LAC and the reference values between $55 \mathrm{keV}$ and $65 \mathrm{keV}$. We suspect that the detector resolution is lower for the higher flux flat field and at places in the spectrum of higher discontinuities the LAC is affected.

The data is rebinned in low- and high energy. The formulation presented here for the thresholds is compatible with an arbitrary number of bins. As an example, merging energyresolved data in the optimized bi-energy bins approach defined in the subsection 3.2 is performed by summing the corrected incident photon flux $I_{k}$ for selected $k$ energy bins before converting to line integrals as:

$$
I_{L}=\sum_{k=\mathrm{i}}^{k=b_{1}} I_{k}, \quad I_{H}=\sum_{k=b_{2}}^{k=\mathrm{f}} I_{k}
$$

where $I_{L}$ and $I_{H}$ are merged photon flux for low- and highenergies, and $\mathrm{i}$ and $\mathrm{f}$ denote low- and high-energy threshold bins, respectively. $b_{1}$ and $b_{2}$ are the upper and lower thresholds of low- and high-energies, respectively (in Fig. 1).

The classification performance has been tested for optimized bi-, 6, 15, 30, 45 and 90 energy bins and the results are presented in the subsection 3.4. Titanium showed the LAC noticeably deviated from the reference values at lower energies that is due to complete attenuation of radiation at this energy range. Fig. 6 presents the measured, fitted and reference LAC of titanium. The deviated LAC of titanium has been fitted by using polynomial extrapolation with MATLAB ${ }^{\circledR}$ 's 

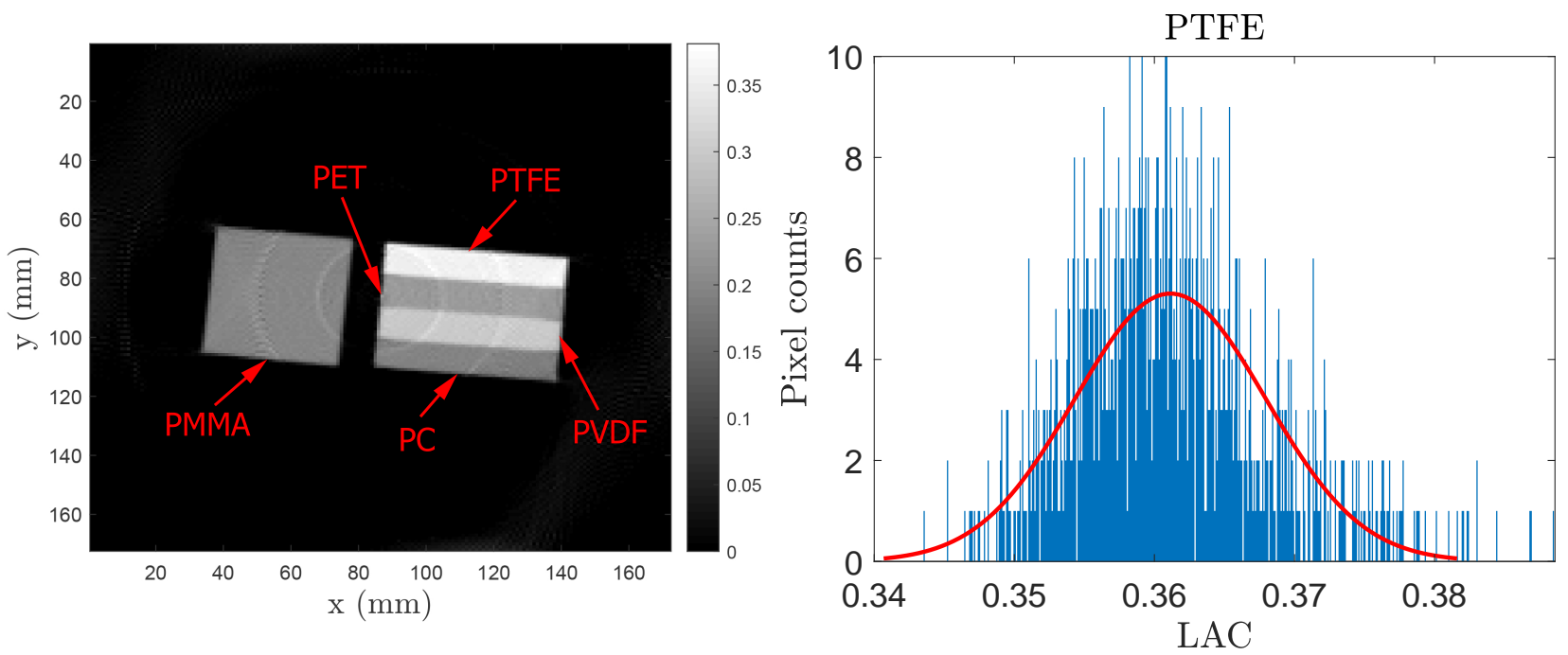

Figure 4: The mean LAC extraction based on the normal distribution. Left: 2D reconstruction of a sample including the five plastic materials indicated by red arrows. The color bar shows linear attenuation coefficients (LACs) for the low energy bin of the optimized bi-energy approach defined in the subsection 3.2. Right: the histogram of PTFE plastic (blue bars) shown with a Gaussian peak fit (red line). The pixel counts represent the number of pixels in the PTFE plastic area marked by the arrow to the left, each having the corresponding LACs presented on the $\mathrm{x}$-axis.

polyfit function between $32.1 \mathrm{keV}$ and $131.3 \mathrm{keV}$, which became low- and high-energy thresholds, respectively for 15 , 30,45 and 90 energy bins approaches and for all materials. The energy bins in these approaches were spaced uniformly between low- and high-energy thresholds with equal width bins. For optimized bi- and 6 energy bins approaches the number of bins was insufficient for the extrapolation, instead the deviated LAC of titanium from $32.1 \mathrm{keV}$ was truncated and the energy bins were spaced over $57.5 \mathrm{keV}$ and 131.3 $\mathrm{keV}$ that were set as low- and high-energy thresholds, respectively for all materials. Without LAC extrapolation, the number of bins in this PCD is insufficient to employ 90 bins for classifications between $57.5 \mathrm{keV}$ and $131.3 \mathrm{keV}$ thresholds as shown in Fig. 5.

Fig. 6 also shows counts for flat field spectra and low- and high-energy thresholds for all variations of energy bins used and the gap between low- and high-energies in the optimized bi-energy bins approach.

\subsection{Calibration of Basis functions and $n$}

From Eq. (9), LAC for each energy bin can be expressed as

$$
\mu_{m}\left(E_{k}\right)=\rho_{e, m}\left(Z_{\text {eff }, m}^{n-1} p\left(E_{k}\right)+c\left(E_{k}\right)\right),
$$

where $m=1,2, \ldots, M, k=1,2, \ldots, K$ with $M$ being the total number of reference materials listed in Table 1 and $K$ being the number of energy bins used for a particular classification approach. $p\left(E_{k}\right)$ and $c\left(E_{k}\right)$ are photoelectric absorption and Compton scattering basis functions for energy bin $E_{k}$, respectively. These are calibrated and the same for all materials.

We optimize not only the basis functions, but also the parameter $n$ for better estimation. Due to the non-linearity with respect to $n$, solving Eq. (12) is not straightforward. We impose positivity constraints for all basis functions and $n$ on the solution. With this constraint, we formulate the objective function as follows:

$$
\min _{0 \leq p_{1}, c_{1}, \ldots, p_{K}, c_{K}, n} \sum_{m=1}^{M} \sum_{k=1}^{K}\left(\mu_{m}\left(E_{k}\right)-\rho_{\mathrm{e}, m}\left(Z_{\mathrm{eff}, m}^{n-1} p_{k}+c_{k}\right)\right)^{2},
$$

where $p_{k}=p\left(E_{k}\right)$ and $c_{k}=c\left(E_{k}\right)$. To optimize Eq. (13), we employ a nonlinear least square solver based on trust region method [53]. The minimization was performed with MATLAB ${ }^{\circledR}$ 's Isqnonlin function with initial constraints that we chose as $p_{k}^{0}=0.5, c_{k}^{0}=0.5$ and $n^{0}=3.6$ for all $k$. The calibration results showed no particular dependency on the starting values.

At low energies, the photoelectric cross section is a strong function of atomic number, as seen in Eq. (1) and Eq. (3). This effect can be used to achieve strong contrast even for materials with small differences in electron densities and effective atomic numbers. For most biological materials, as the photon energy increases over $60 \mathrm{keV}$, the dependence of the LACs on atomic number rapidly decreases and the photon attenuation occur mostly due to Compton interaction where mass density and electron density dominate the LACs [43]. Fig. 7 shows the resulting optimized values of the $p\left(E_{k}\right), c\left(E_{k}\right)$ basis functions and of $n$ for all the multiple energy bins approaches analyzed in our work. As expected, over roughly $60 \mathrm{keV}$, photoelectric absorption basis functions that decrease over the whole energy range become lower than those for Compton scattering part. As a result, the $p\left(E_{k}\right)$ basis functions values in optimized bi- and 6 energy bins approaches are 1.7 to 5 and 3.5 to 19.7 times lower than $c\left(E_{k}\right)$, respectively.

The reported values of exponent $n$ defined in photo- 

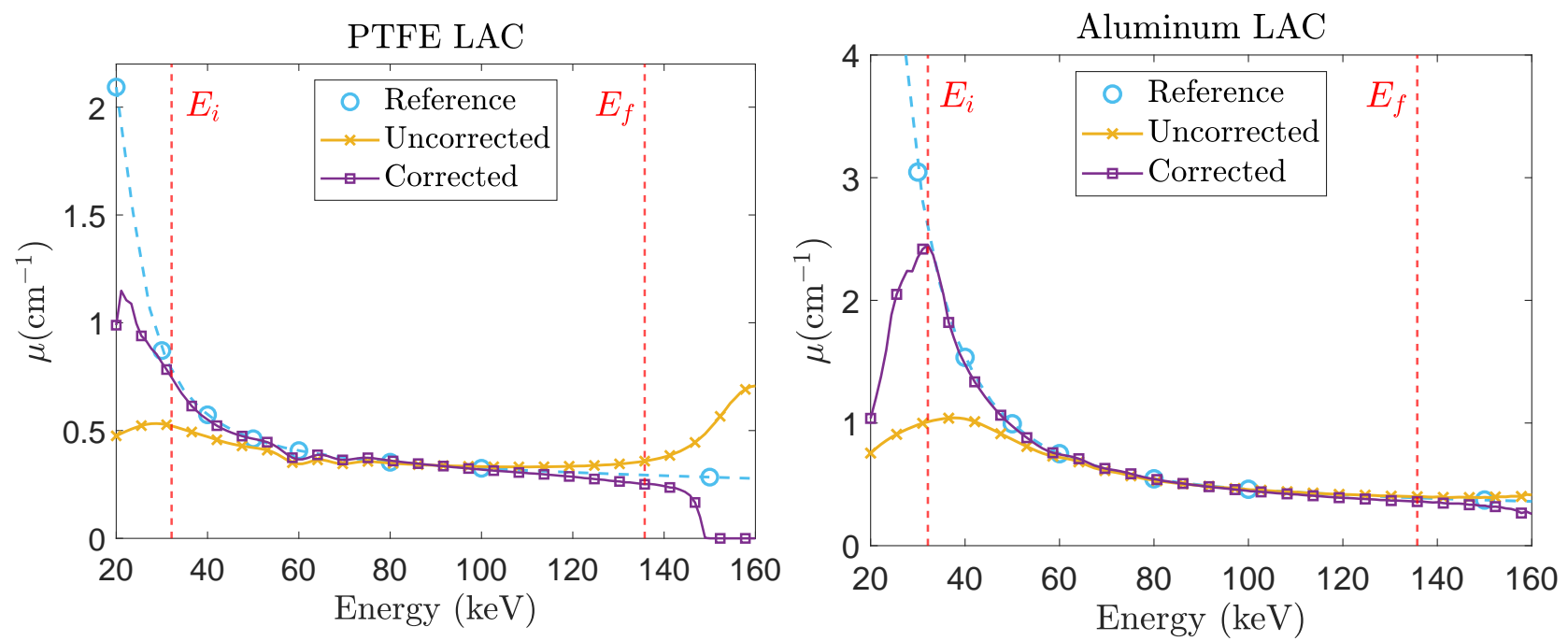

Figure 5: Energy resolved LAC for PTFE (left) and aluminum (right) obtained with and without spectral correction. The reference LACs were defined by using NIST cross-sections, [52]. Where the LAC deviates at low- and high-energies the spectra are truncated, and low- and high-energy thresholds, $E_{\mathrm{i}}$ and $E_{\mathrm{f}}$ are constant for all materials as shown with the vertical black dash-dotted lines.
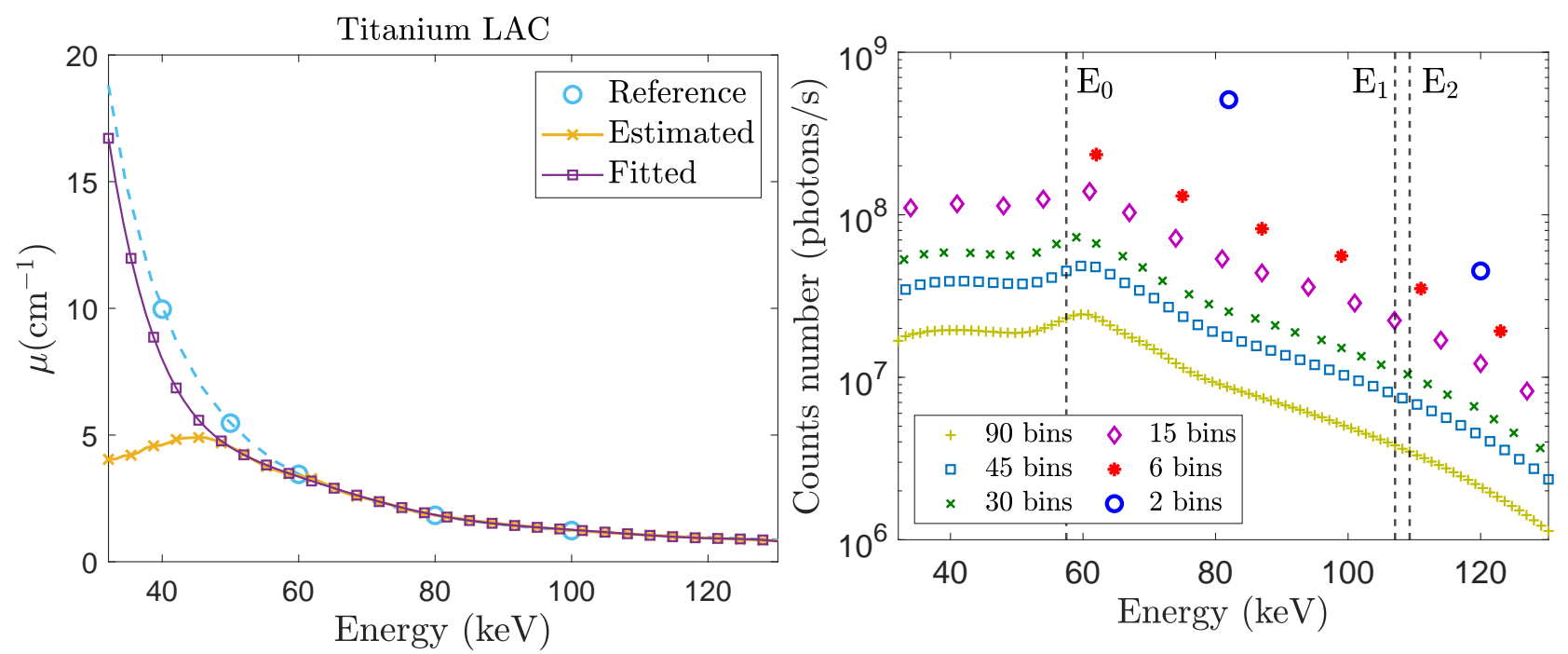

Figure 6: Fitting LAC by extrapolation and photon counts for all the approaches for variations of energy bins used. Left: the measured and fitted LAC obtained by using polynomial extrapolation and the reference LAC of titanium. Right: counts in the flat field spectra for optimized bi-, 6, 15, 30, 45 and 90 energy bins approaches. A vertical lines at $E_{0}(57.5 \mathrm{keV})$ is the low-energy threshold for optimized bi- and 6 energy bins, and the lines at $E_{1}(107 \mathrm{keV})$ and $E_{2}(109.3 \mathrm{keV})$ shows the gap between lowand high-energies of optimized bi-energy bins. Note logarithmic scale on the y-axis.

electric cross section term in the literature changes between nearly 3.94 and 5 , as a function of the photon energy and also materials [37, 42-45, 47, 54]. Weber et al.(1969) [42] proposed the value of 4.4 for $n$. For CT systems, the suggested exponents were 3.94 by Phelps et al. (1975) [55], 4.4 by Cho et al. (1975) [43], 4.62 by Rutherford et al. (1976) [54] and 4.8 by McCullough (1975) [41]. For idealized dual-energy CT imaging in the energies between 20 $\mathrm{keV}$ and $1000 \mathrm{keV}$, Williamson et al. (2006) [45] separated parameterization into two sets of exponents depending on atomic number range and found 5.05 and 4.69 for $2 \leq Z \leq 8$ and $8<Z \leq 20$, respectively. They esti- mated theoretically linear attenuation coefficients with absolute mean and maximum accuracy errors of up to $2.2 \%$ and $6 \%$, respectively, however there were relatively higher errors at low energies and at higher $Z$. For high-energy $\mathrm{x}$-ray cargo inspection, Langeveld (2017) [47] gave an improved Lehmann-type parameterization of LACs at energies of $20-1000 \mathrm{keV}$, which resulted in a better definition of photoelectric absorption cross section term and in turn $Z_{\text {eff }}$. $X$-ray attenuation coefficients were fitted quite well over a broad range of $Z$. The value of $n$ in their fits was 4.14. In our work, the optimized values of exponent $n$ were found to be between 3.60 and 4.09 depending on the number of en- 

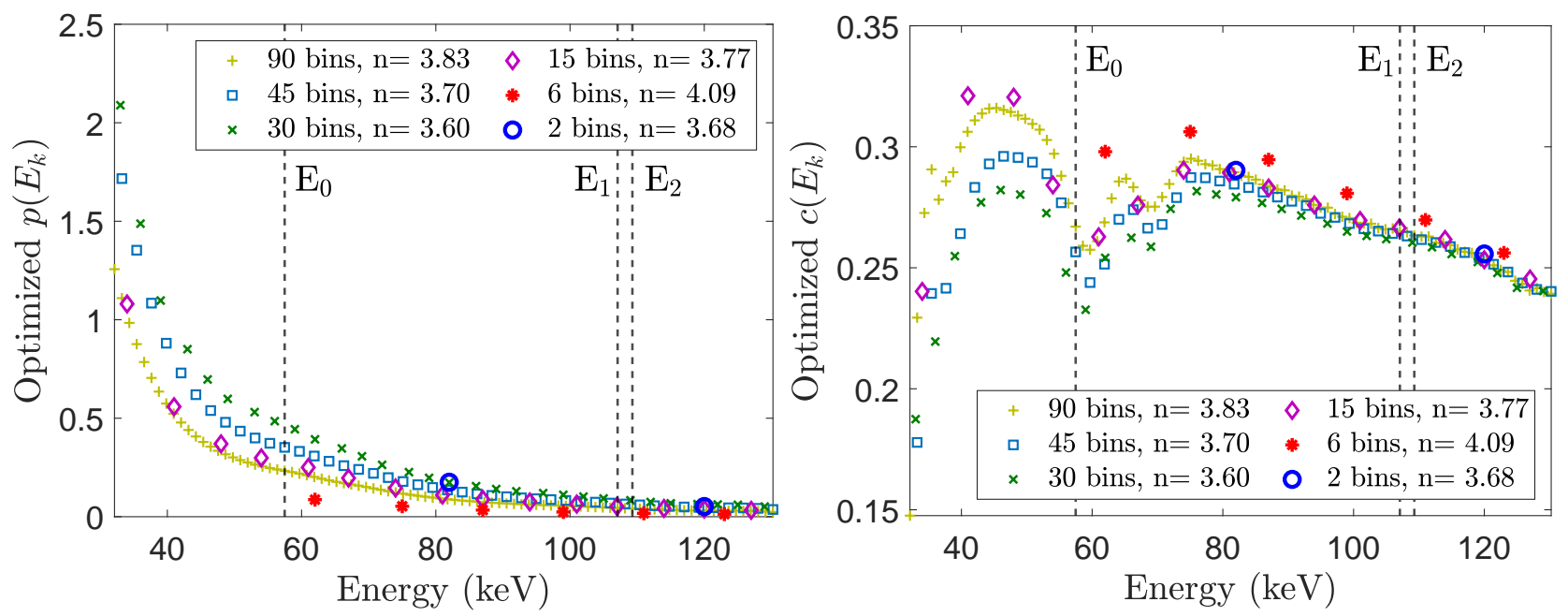

Figure 7: Optimized photoelectric absorption $p\left(E_{k}\right)$ (left) and Compton scattering $c\left(E_{k}\right)$ (right) basis functions for optimized bi-, $6,15,30,45$ and 90 energy bins approaches. The optimized values of exponent $n$ are also presented for each multiple energy bins approach. A vertical lines at $E_{0}(57.5 \mathrm{keV})$ is the low-energy threshold for optimized bi- and 6 energy bins, and the lines at $E_{1}(107 \mathrm{keV})$ and $E_{2}(109.3 \mathrm{keV})$ shows the gap between low- and high-energies of optimized bi-energy bins.

ergy bins, as shown in Fig. 7. The higher the $n$ value is, the lower $p\left(E_{k}\right)$ and the higher $c\left(E_{k}\right)$ basis functions are for all energies. The highest value of $n=4.09$ found for the approach using 6 energy bins presents the lowest classification performance, as discussed in the subsection 3.4. The reason for relatively lower values of $n$ found in our work may be due to our parameterization and the multi-spectral dimensionality of LACs defined in Eq. (12).

\section{6. $\left(\rho_{\mathrm{e}}, Z_{\text {eff }}\right)$ Calculation}

The calibration parameters $\left(p\left(E_{k}\right), c\left(E_{k}\right)\right.$ and $\left.n\right)$ and the measured LAC $\mu\left(E_{k}\right)$ are used to retrieve $\left(\rho_{\mathrm{e}}, Z_{\text {eff }}\right)$. For $M$ - and $K$ - total number of unknown materials and energy bins, respectively, we reformulate Eq. (12) as a linear system equation as follows:

$$
\left(\begin{array}{ccccc}
\vec{p} & \vec{c} & & & \\
& & \ddots & & \\
& & & \vec{p} & \vec{c}
\end{array}\right)\left(\begin{array}{c}
z_{1} \\
\rho_{\mathrm{e}, 1} \\
\vdots \\
z_{M} \\
\rho_{\mathrm{e}, M}
\end{array}\right)=\left(\begin{array}{c}
\vec{\mu}_{1} \\
\vdots \\
\vec{\mu}_{M}
\end{array}\right)
$$

where $z_{m}$ is a temporary variable introduced instead of $\rho_{\mathrm{e}, m} Z_{\text {eff }}^{n-1}$ and $\vec{p}=\left(p_{1}, \ldots, p_{K}\right)^{T}, \vec{c}=\left(c_{1}, \ldots, c_{K}\right)^{T}$ and $\vec{\mu}_{m}=\left(\mu_{m}\left(E_{1}\right), \ldots, \mu_{m}\left(E_{K}\right)\right)^{T}$. In the latter equation, the size of the linear matrix is $(M \times K)$-by- $(2 \times M)$ and the number of unknowns is $2 \times M$. We use a linear least square solver called lsqnonneg function in MATLAB ${ }^{\circledR}$ with the positivity constraint on the solution returning the vector $\left(z_{1}, \rho_{\mathrm{e}, 1}, \ldots, z_{M}, \rho_{\mathrm{e}, M}\right)$ that minimizes the norm. After solving it, the effective atomic numbers are retrieved for all materials by

$$
Z_{m}=\left(\frac{z_{m}}{\rho_{\mathrm{e}, m}}\right)^{\frac{1}{n-1}} .
$$

The method accuracy was estimated as the percent relative deviation from the reference values for $\rho_{\mathrm{e}}$ and $Z_{\text {eff }}$ as:

$$
\begin{gathered}
\Delta Z_{\text {eff }}^{\text {rel }}=100 \% \cdot \frac{Z_{\text {eff }}^{\text {est }}-Z_{\text {eff }}^{\text {ref }}}{Z_{\text {eff }}^{\text {ref }}}, \\
\Delta \rho_{\mathrm{e}}^{\text {rel }}=100 \% \cdot \frac{\rho_{\mathrm{e}}^{\text {est }}-\rho_{\mathrm{e}}^{\text {ref }}}{\rho_{\mathrm{e}}^{\text {ref }}}
\end{gathered}
$$

where superscripts est and ref refer to the estimated and reference values, respectively.

\section{Results and Discussions}

\subsection{Investigating classification performance as a function of exponent $l$}

We studied how the classification performance changes with the selection of the exponent $l$ defined in $Z_{\text {eff }}$ formula (Eq. (8) ) for the materials such as plastics and liquids listed in Table 1, to tune this value on the best match for our experimental data. The classifications were performed using plastic materials as references in the calibration step and optimized bi-energy bins for the calculations. Fig. 8 presents the dependence of relative errors for $Z_{\text {eff }}$ on exponent $l$ for the materials shown. Based on the mean relative deviations, we find that the optimal range of exponent $l$ is between 7.2 and 10.5 with lower than $1 \%$ deviations and the minimum deviation is approximately $0.9 \%$ at $l=8.0$ whereas the deviation is $1.4 \%$ at the reference value of $l=3.8$. Thus, the exponent $l$ given in the references is not optimal with this set of materials, source spectrum and detector response. To preserve consistence with the previously published works in comparing the performance, we keep the reference value of $l=3.8$ for our calculations of reference $Z_{\text {eff }}$ values. 



Figure 8: The variation of accuracy with exponent $l$. Left: the percent relative deviations for $Z_{\text {eff }}$ defined in Eq. (8), as a function of exponent $l$ for the materials presented. Right: the mean percent relative deviation for $Z_{\text {eff }}$ calculated from absolute values of relative deviations for each material shown in the left frame. As expected, the relative deviations for the electron density for all the materials did not show noticeable dependence on $l$.

\subsection{Optimizing the method for bi-energy bins}

Fig. 1 illustrates the gap between low- and high-energies when using optimized bi-energy bins. How low and high energy photons are binned together is important for classification performance. Therefore, to optimize the method the gap is shifted from low- to high-energy threshold in increments of one energy bin and the gap width is selected as two energy bins equal to $2.3 \mathrm{keV}$ and kept constant over the whole energy range. Fig. 9 presents how the photon counts for low- and high-energies change with respect to position of the gap corresponding to the upper and lower thresholds of low- and high-energies, respectively.

Fig. 10 shows the changes in the percent relative deviations for $\rho_{\mathrm{e}}$ and $Z_{\text {eff }}$ of the mean values (for all materials), PMMA, water, aluminum and titanium between the low- and high-energy thresholds when using bi-energy bins. The chart shows that the relative deviations for the materials with higher $Z_{\text {eff }}$ values such as aluminum and titanium remain lower and more stable over the whole energy range compared to liquids and plastics for both $\rho_{\mathrm{e}}$ and $Z_{\text {eff }}$. The optimal classification performance is reached when lowenergy is between $57.5 \mathrm{keV}$ and $107 \mathrm{keV}$ while high-energy is $109.3 \mathrm{keV}$ to $131.3 \mathrm{keV}$ with mean relative deviations of $1.5 \%$ and $3.1 \%$ for $\rho_{\mathrm{e}}$ and $Z_{\text {eff }}$, respectively. The results for all the materials obtained with optimized bi-energy bins are presented in the next subsection. In contrast to a simulation study presented by Wang et al. [26] estimating the thickness of the two basis materials, we find that similar incident photon flux in low- and high-energies when using bi-energy bins do not provide the optimal classification performance, this result is seen in Fig. 9. The reason may be because our results are based on experimental data with realistic noise level and spectral artefact.

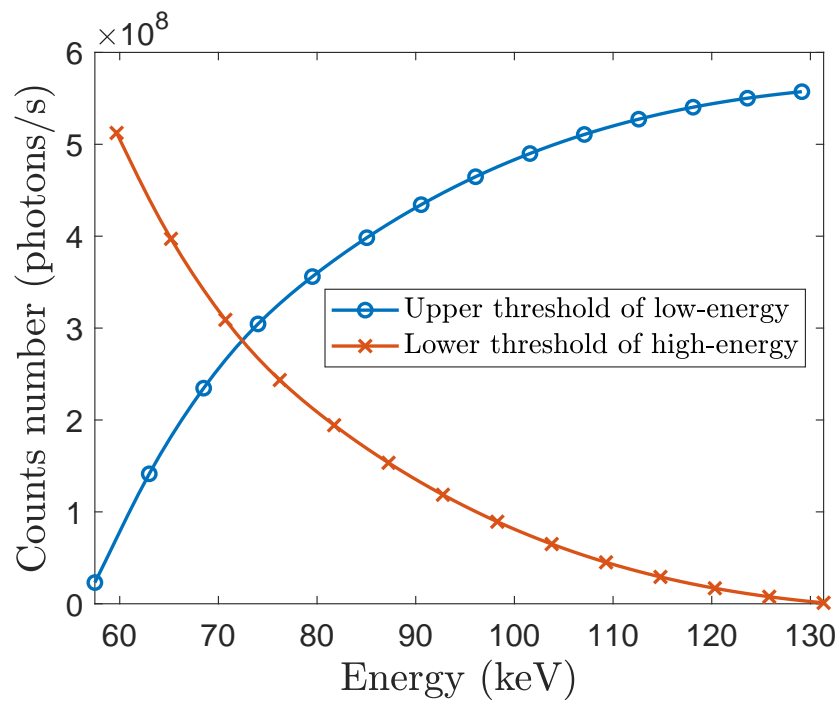

Figure 9: Photon counts of low- and high-energies of all detector pixels in the flat field spectrum as a function of the gap position being shifted from low- to high-energy threshold. The gap corresponds to the upper and lower thresholds of low- and high-energies in the bi-energy bins approach, respectively.

\section{3. $\left(\rho_{\mathrm{e}}, Z_{\text {eff }}\right)$ map}

Fig. 11 shows a $\left(\rho_{\mathrm{e}}, Z_{\text {eff }}\right)$ chart of estimated and reference values. Table 2 lists the percent relative deviations for both of estimated material features for SIMCAD and SRZE methods. The SIMCAD method gives promising results with mean relative deviations of $1.5 \%$ for $\rho_{\mathrm{e}}$, and $3.1 \%$ for $Z_{\text {eff }}$ using optimized bi-energy bins, whereas the SRZE method yields deviations of $2.4 \%$ and $1.9 \%$ respectively with 64 energy bins [28]. With only two energy bins, the SRZE method has a noticeably higher deviation of $9.4 \%$ for $\rho_{\mathrm{e}}$, and $13.4 \%$ for $Z_{\text {eff }}$ for single material samples [32]. Thus, our 

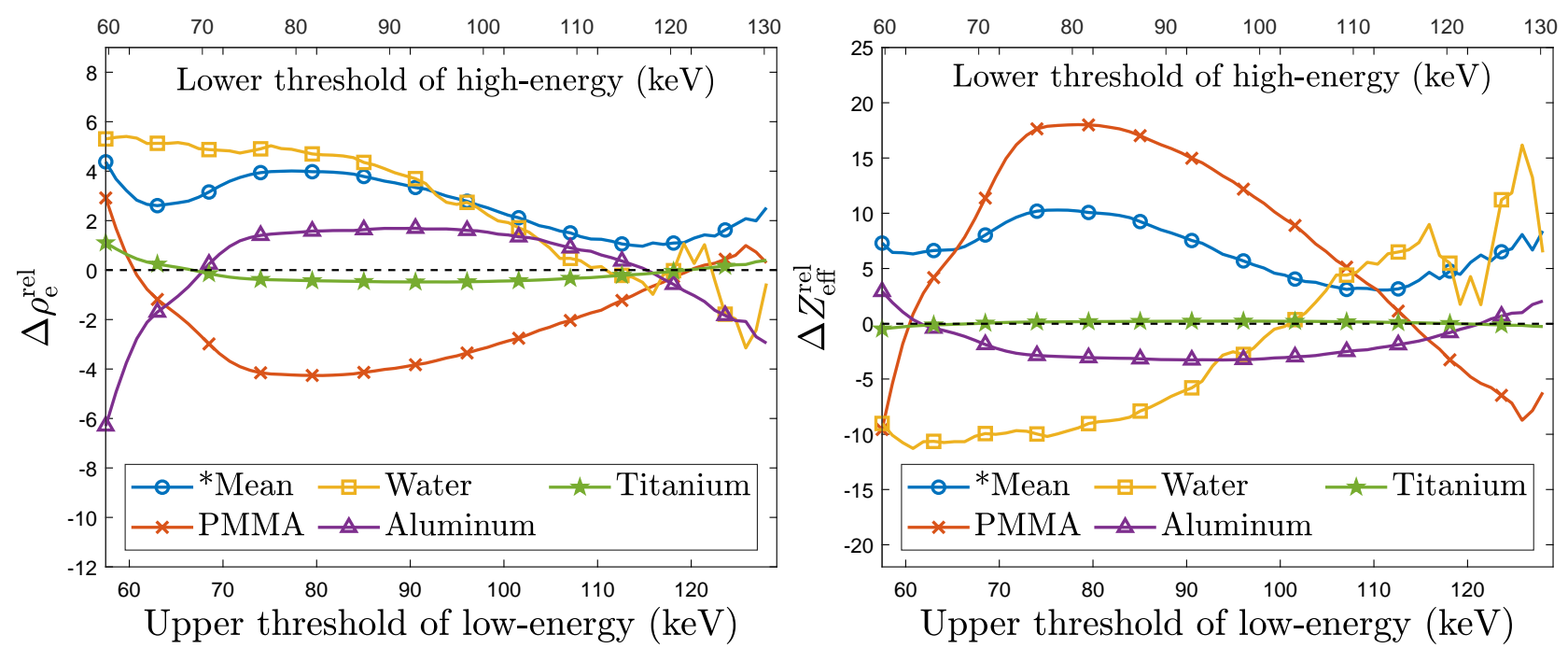

Figure 10: Optimization of the bi-energy-bin method based on relative deviations for $\rho_{\mathrm{e}}$ (left) and $Z_{\text {eff }}$ (right). ${ }^{*} T h e$ mean deviation was calculated from the absolute values of relative deviations for each material listed in Table 1.
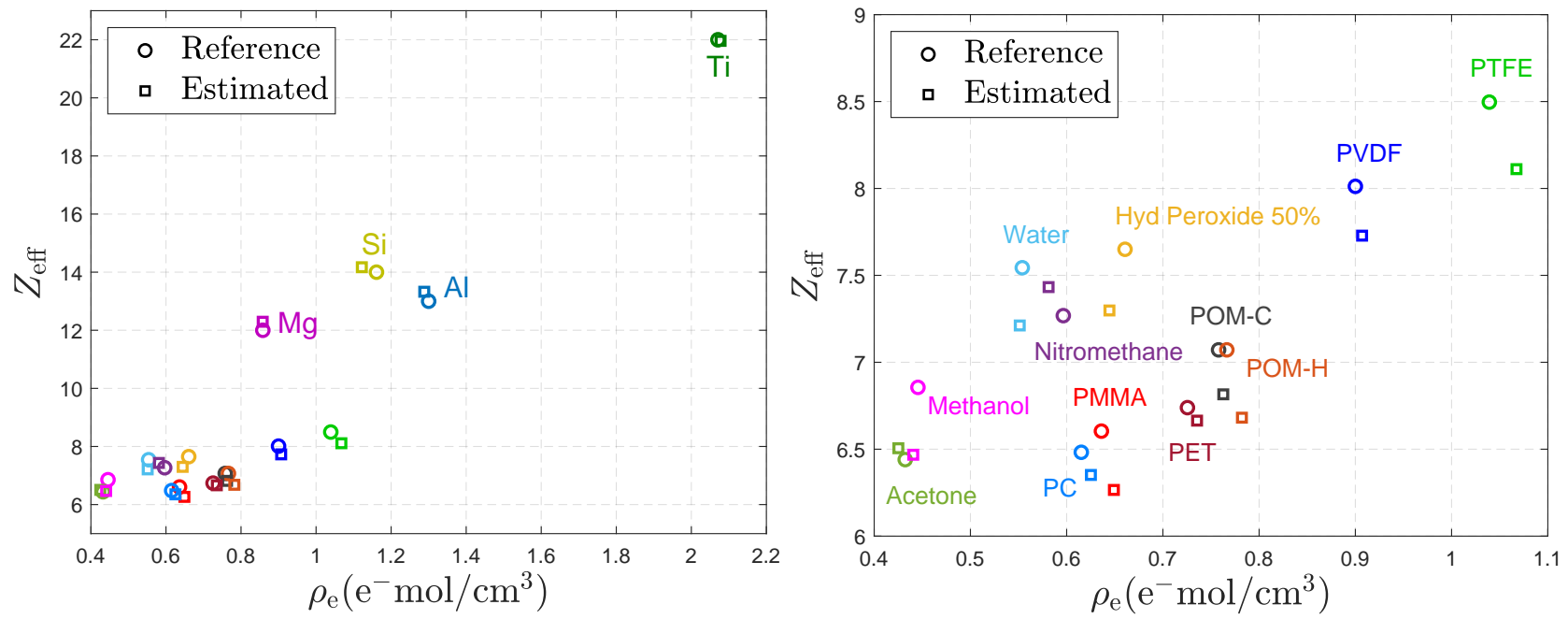

Figure 11: Estimated and reference $\left(\rho_{\mathrm{e}}, Z_{\mathrm{eff}}\right)$ obtained with the SIMCAD method using optimized bi-energy bins. A magnified version of the lower left part of the left frame is presented in the right frame to avoid overlap of the material labelled names.

method using much fewer energy bins can provide a significant reduction in computing time, which is important for security screening scanners. It can be noted from the table that as the effective atomic number increases, the classification accuracy also increases. Thus the method may be robust also for materials with higher $Z_{\text {eff }}$ values.

\subsection{Classification performance as a function of the number of energy bins used}

How a source spectrum is sampled for all the multiple energy bins approaches is described in the subsection 2.4. Fig. 12 highlights the relative errors for $\left(\rho_{\mathrm{e}}, Z_{\text {eff }}\right)$ depending on the number of energy bins used. For $\rho_{\mathrm{e}}$, the mean relative error values increase as the number of energy bins used increases reaching $4.2 \%$ at 90 energy bins. For $Z_{\text {eff }}$, the error remains almost unchanged being equal to $3.3 \%$ at 90 energy bins, with an exception for 6 energy bins where the error is $7.2 \%$. Materials with higher $Z_{\text {eff }}$ values such as aluminum and titanium show noticeably lower relative error for all numbers of energy bins used compared to plastics and liquids.

The classification performance is worse for the 6 energy bins approach compared to optimized-bi-energy bins. The reason may be that the thresholds of 6 energy bins approach are not optimized whereas those for optimized-bienergy bins are. Thus, optimizing the energy bin thresholds is desired for better performance when the number of energy bins used is low. A similar phenomenon was previously observed in radiography studies by Beldjoudi et al. [20, 25]. However, contrary to their findings, employing 15, 30, 45 and 90 energy bins does not enhance but rather deteriorates classification performance relative to optimized-bi-energy bins approach. This may be because energy bin widths for $15,30,45$ and 90 energy bins, which are equally distributed throughout the spectrum are below the energy resolution of 
Table 2

The percent relative deviations for $\left(\rho_{\mathrm{e}}, Z_{\text {eff }}\right)$ with the reference values. To compare, SRZE estimations are taken from [28, 32]. *The mean values were calculated from absolute values of each column.

\begin{tabular}{|c|c|c|c|c|c|c|}
\hline Material & $\rho_{\mathrm{e}}^{\mathrm{ref}}\left(\mathrm{e}^{-} \mathrm{mol} / \mathrm{cm}^{3}\right)$ & $\begin{array}{l}\text { SIMCAD } \\
\Delta \rho_{\mathrm{e}}^{\text {rel }}(\%)\end{array}$ & $\begin{array}{l}\text { SRZE } \\
\Delta \rho_{\mathrm{e}}^{\mathrm{rel}}(\%)\end{array}$ & $Z_{\text {eff }}^{\text {ref }}$ & $\begin{array}{l}\text { SIMCAD } \\
\Delta Z_{\text {eff }}^{\text {rel }}(\%)\end{array}$ & $\begin{array}{l}\text { SRZE } \\
\Delta Z_{\text {eff }}^{\text {rel }}(\%)\end{array}$ \\
\hline PMMA & 0.636 & -2.0 & -2.3 & 6.60 & 5.1 & -0.3 \\
\hline PTFE & 1.039 & -2.7 & 0.1 & 8.50 & 4.5 & -2.5 \\
\hline PVDF & 0.9 & -0.8 & -0.8 & 8.01 & 3.5 & -1.4 \\
\hline PC & 0.615 & -1.6 & 1.4 & 6.48 & 2.0 & -1.8 \\
\hline POM-C & 0.758 & -0.6 & -1.4 & 7.07 & 3.6 & -0.9 \\
\hline PET & 0.726 & -1.4 & -3.1 & 6.74 & 1.1 & -0.1 \\
\hline POM-H & 0.766 & -2.0 & -2.4 & 7.07 & 5.5 & -0.4 \\
\hline Acetone & 0.432 & 1.6 & -2.3 & 6.44 & -1.0 & 5.0 \\
\hline $\mathrm{H}_{2} \mathrm{O}_{2}(50 \%)$ & 0.661 & 2.4 & -0.2 & 7.65 & 4.6 & 5.4 \\
\hline Methanol & 0.446 & 1.1 & 1.4 & 6.86 & 5.6 & -4.7 \\
\hline Water & 0.554 & 0.5 & -6.9 & 7.54 & 4.4 & -2.7 \\
\hline Nitromethane & 0.597 & 2.6 & 1.7 & 7.27 & -2.3 & 2.5 \\
\hline Aluminum & 1.3 & 0.9 & -3.2 & 13 & -2.5 & 0.7 \\
\hline Silicon & 1.161 & 3.4 & -5.5 & 14 & -1.2 & -0.7 \\
\hline Magnesium & 0.858 & 0.03 & -5.4 & 12 & -2.4 & 0.8 \\
\hline Titanium & 2.071 & -0.3 & -0.7 & 22 & 0.2 & -1.2 \\
\hline *Mean & - & 1.5 & 2.4 & - & 3.1 & 1.9 \\
\hline
\end{tabular}

the detector ( $8 \mathrm{keV}$ ) corresponding, respectively, to energy bin widths of $6.6 \mathrm{keV}, 3.3 \mathrm{keV}, 2.2 \mathrm{keV}$ and $1.1 \mathrm{keV}$; Secondly the detector noise becomes greater as the number of energy bins increases. Since the bin width for approaches using 30 and 45 energy bins are smaller compared to the approach of 15 energy bins, they are less dependent on energy threshold optimization. The approach of 90 energy bins does not require any threshold optimization. Despite this fact, 15 energy bins approach without the energy bin threshold optimization appears to have better classification performance compared to 30, 45 and 90 energy bins. Overall optimized bi-energy bins achieve the best classification performance, and shows the best robustness for all the materials we classified in this work.

Fig. 13 shows the mean relative deviations obtained by corrected and uncorrected LACs from optimized bi-energy bins, which equals $5.0 \%$ and $37.9 \%$ for $\rho_{\mathrm{e}}$ and $Z_{\text {eff }}$, respectively without the correction. Therefore the method requires the use of a spectral correction algorithm.

\section{Conclusion}

We have presented the SIMCAD method for material characterization from system-independent physical parameters $\left(\rho_{\mathrm{e}}, Z_{\text {eff }}\right)$ using Spectral CT systems. We explored the influence of the number of used energy bins on the performance of the method by applying different energy thresholds to define the energy bins. Employing a non-optimized energy bins approaches with a low number of energy bins used leads to a noticeable decrease in classification performance. It was presented that the approaches with energy bin widths below the detector energy resolution does not provide noticeable enhancement in classifications further, and we found the method to be most accurate employing just optimized bi-energy bins. The formulation of method however, is compatible with an arbitrary number of energy bins. We saw the use of a correction algorithm remove most detector response artefacts from the LAC, and classification even with optimized bi-energy bins is shown to require the correction. SIMCAD requires a calibration step using a set of reference materials to calibrate photoelectric absorption and Compton scattering basis functions and $n$ from the LAC expression.

In contrast to the SRZE method, which reaches the best accuracy with 64 energy bins [28], the SIMCAD method gives comparable accuracy with only optimized bi-energy bins, which gives up to 32 times reduction in computing time due to the reduced number of CT reconstructions required. This makes the method compatible with the requirements of high speed security scanners. Our method employing a single scan is also comparable to the state-of-the-art techniques using dual-energy CT scans in terms of reconstruction time and material classifications, and it provides a better classification performance for highly attenuating materials $[4,16]$. The method is shown to be applicable in the range of $6 \leq Z_{\text {eff }} \leq 23$ however, since higher $Z_{\text {eff }}$ materials showed noticeably better accuracy compared to plastics and liquids, the method may be expected to be suitable for a larger range of $Z_{\text {eff }}$ materials. Contrary to the results obtained in the studies by Rinkel et al. [24] and Beldjoudi et al. [25], the method gives better accuracy with optimized bi-energy bins, compared to the same technique with 90 energy bins. This may be because the high and low energy thresholds of our technique truncates the LAC's where they have the highest amount of experimental artefacts; Secondly compared to dual energy detectors our energy bins are not 

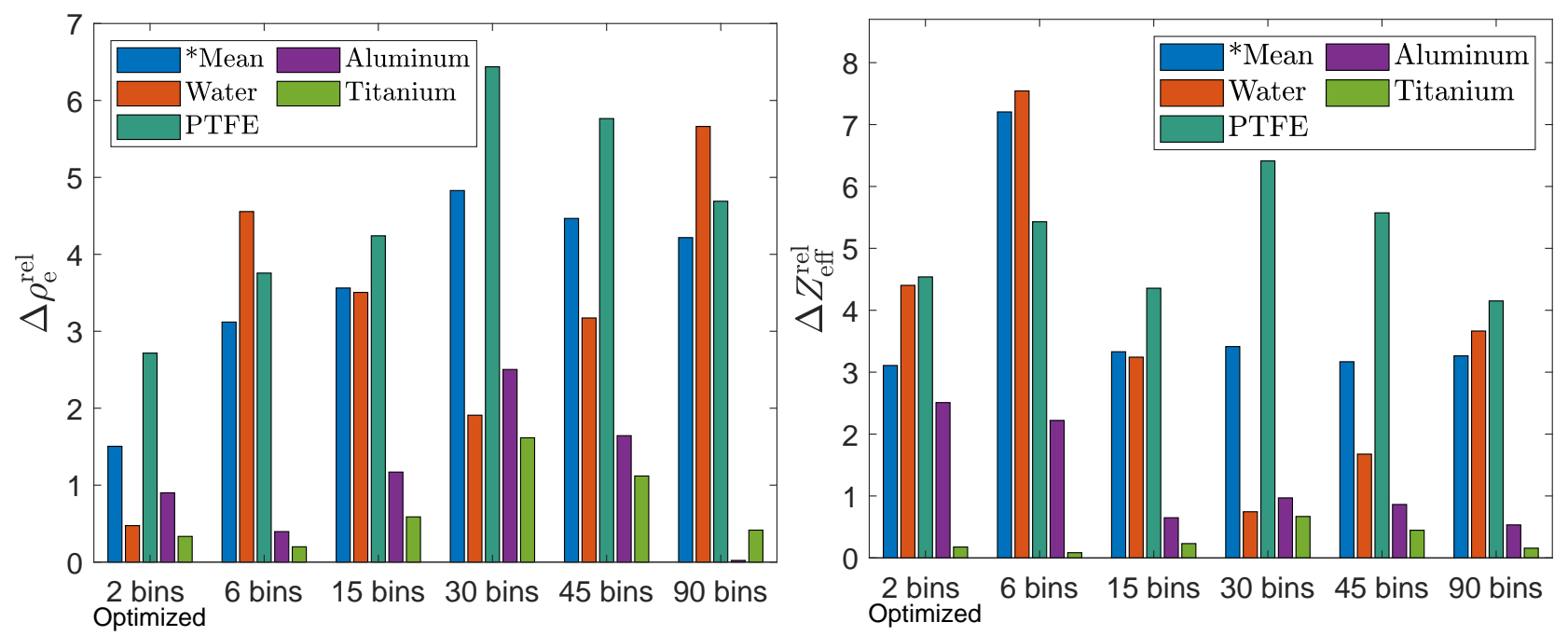

Figure 12: The percent relative deviations for $\rho_{\mathrm{e}}$ (left) and $Z_{\text {eff }}$ (right) as a function of the number of energy bins used for classifications. Optimized bi-energy bins gives better classification performance. *The mean deviation was calculated from absolute values of relative deviations for each material listed in Table 1.

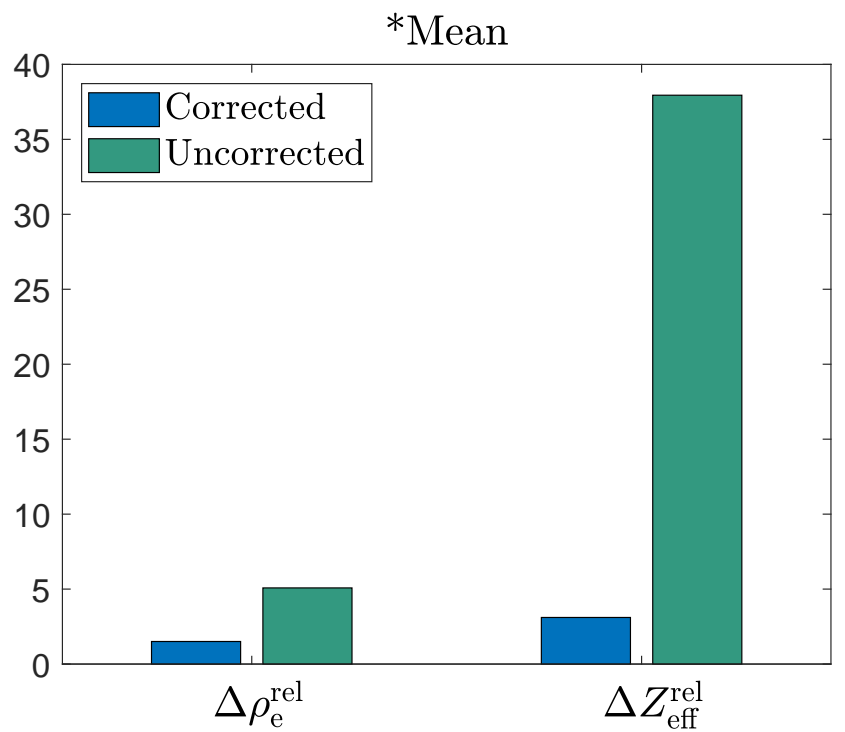

Figure 13: *The mean deviation for $\left(\rho_{\mathrm{e}}, Z_{\mathrm{eff}}\right)$ obtained by corrected and uncorrected LACs in the optimized bi-energy approach, calculated from absolute values of relative deviations for each material used.

overlapping and are thus not influenced by system or sample parameters. The optimized bi-energy approach truncated the low-energy bin below $57.5 \mathrm{keV}$ and therefore the method is expected also to work for the materials with $Z_{\text {eff }} \leq 68$, which do not exhibit absorption edges above this low-energy threshold. This range of atomic numbers include most of the materials scanned by security screening instruments. For further experiments, we will investigate robustness of the technique for a broader range of $Z_{\mathrm{eff}}$, and in the presence of K-edges.

\section{CRediT authorship contribution statement}

Doniyor Jumanazarov: Conceptualization, Methodology, Software, Formal analysis, Investigation, Data curation, Writing - original draft, Visualization. Jakeoung Koo: Software, Validation, Formal analysis. Matteo Busi: Validation, Resources, Writing - review and editing. Henning F. Poulsen: Writing - review and editing, Supervision . Ulrik L. Olsen: Validation, Resources, Data curation, Writing - review and editing, Supervision . Mihai IOVEA: Conceptualization, Methodology, Validation, Writing - review and editing, Supervision, Funding acquisition.

\section{Acknowledgements}

This project has received funding from the European Union Horizon 2020 research and innovation programme under the Marie Skłodowska-Curie grant agreement No. 765604 and it is part of the MUltiscale, Multimodal and Multidimensional imaging for EngineeRING project (MUMMERING Innovative Training Network, www. mummering. eu).

The authors want to acknowledge also the 3D Imaging Center at DTU, where the experiments have been conducted.

\section{References}

[1] M. Ellenbogen, R. Bijjani, Liquids and homemade explosive detection, in: Optics and Photonics in Global Homeland Security V and Biometric Technology for Human Identification VI, volume 7306, International Society for Optics and Photonics, 2009, p. 73060Y. doi:10.1117/12.821806.

[2] H. E. Martz, C. M. Logan, D. J. Schneberk, P. J. Shull, X-ray Imaging: fundamentals, industrial techniques and applications, CRC Press, 2016.

[3] R. E. Alvarez, A. Macovski, Energy-selective reconstructions in Xray computerised tomography, Physics in Medicine and Biology 21 (1976) 733-744. 
[4] S. G. Azevedo, H. E. Martz, M. B. Aufderheide, W. D. Brown, K. M. Champley, J. S. Kallman, G. P. Roberson, D. Schneberk, I. M. Seetho, J. A. Smith, System-Independent Characterization of Materials Using Dual-Energy Computed Tomography, IEEE Transactions on Nuclear Science 63 (2016) 341-350.

[5] Z. Ying, R. Naidu, C. R. Crawford, Dual energy computed tomography for explosive detection, Journal of X-Ray Science and Technology 14 (2006) 235-256.

[6] E. Kalender, Willi A and Perman, WH and Vetter, JR and Klotz, Evaluation of a prototype dual-energy computed tomographic apparatus. I. Phantom studies, Medical physics 13 (1986) 334-339.

[7] C. Robert-Coutant, V. Moulin, R. Sauze, P. Rizo, J. M. Casagrande, Estimation of the matrix attenuation in heterogeneous radioactive waste drums using dual-energy computed tomography, Nuclear Instruments and Methods in Physics Research, Section A: Accelerators, Spectrometers, Detectors and Associated Equipment 422 (1999) 949956.

[8] D. Braz, R. T. Lopes, L. M. Motta, Dual-energy computerized tomography in compacted soil, Geotechnical and Geological Engineering 18 (2000) 221-238.

[9] B. J. Heismann, J. Leppert, K. Stierstorfer, Density and atomic number measurements with spectral x-ray attenuation method, Journal of Applied Physics 94 (2003) 2073-2079.

[10] M. Iovea, M. Neagu, O. G. Duliu, G. Mateiasi, High accuracy x-ray dual-energy experiments and non-rotational tomography algorithm for explosives detection technique in luggage control, in: Int. Symp. Digital Industrial Radiology and Computed Tomography, 2007, p. 8.

[11] J. S. Park, J. K. Kim, Calculation of effective atomic number and normal density using a source weighting method in a dual Energy X-ray inspection system, Journal of the Korean Physical Society 59 (2011) 2709-2713.

[12] T. P. Szczykutowicz, Z. Qi, G.-H. Chen, A simple image based method for obtaining electron density and atomic number in dual energy CT, in: Medical Imaging 2011: Physics of Medical Imaging, volume 7961, International Society for Optics and Photonics, 2011, p. 79613A. doi:10.1117/12.878076.

[13] O. Semerci, E. L. Miller, A parametric level-set approach to simultaneous object identification and background reconstruction for dualenergy computed tomography, IEEE Transactions on Image Processing 21 (2012) 2719-2734.

[14] G. Landry, J. Seco, M. Gaudreault, F. Verhaegen, Deriving effective atomic numbers from DECT based on a parameterization of the ratio of high and low linear attenuation coefficients, Physics in Medicine and Biology 58 (2013) 6851-6866.

[15] K. Wells, D. A. Bradley, A Review of X-ray Explosives Detection Techniques for Checked Baggage, Applied Radiation and Isotopes 70 (2012) 1729-1746.

[16] K. M. Champley, S. G. Azevedo, S. Member, I. M. Seetho, S. M. Glenn, L. D. Mcmichael, J. A. Smith, J. S. Kallman, W. D. Brown, H. E. Martz, Method to Extract System-Independent Material Properties From Dual-Energy X-Ray CT, IEEE Transactions on Nuclear Science 66 (2019) 674-686.

[17] P. M. Shikhaliev, Energy-resolved computed tomography: First experimental results, Physics in Medicine and Biology 53 (2008) 55955613.

[18] E. I. Parsai, D. Shvydka, J. Kang, Design and optimization of large area thin-film CdTe detector for radiation therapy imaging applications, Medical Physics 37 (2010) 3980-3994.

[19] A. Brambilla, P. Ouvrier-Buffet, J. Rinkel, G. Gonon, C. Boudou, L. Verger, CdTe linear pixel X-ray detector with enhanced spectrometric performance for high flux X-ray imaging, IEEE Transactions on Nuclear Science 59 (2012) 1552-1558.

[20] G. Beldjoudi, V. Rebuffel, L. Verger, V. Kaftandjian, J. Rinkel, Multidimensional data processing methods for material discrimination using an ideal X-ray spectrometric photon counting detector, IEEE Transactions on Nuclear Science 58 (2011) 3190-3203.

[21] X. Wang, D. Meier, K. Taguchi, D. J. Wagenaar, B. E. Patt, E. C. Frey, Material separation in X-ray CT with energy resolved photon-counting detectors, Medical Physics 38 (2011) 1534-1546.

[22] V. Rebuffel, J. Rinkel, J. Tabary, L. Verger, New perspectives of Xray techniques for explosive detection based on $\mathrm{CdTe} / \mathrm{CdZnTe}$ spectrometric detectors, in: Proc. of the Int. Symp. on Digital Industrial Radiology and Computed Tomography, volume 2, 2011, pp. 1-8.

[23] Limor Martin, Enhanced information extraction in multi-energy Xray tomography for security, Phd thesis, Boston University, 2014.

[24] J. Rinkel, G. Beldjoudi, V. Rebuffel, C. Boudou, P. Ouvrier-buffet, G. Gonon, L. Verger, A. Brambilla, Experimental Evaluation of Material Identification Methods With CdTe X-ray Spectrometric Detector, IEEE Transactions on Nuclear Science 58 (2011) 2371-2377.

[25] G. Beldjoudi, V. Rebuffel, L. Verger, V. Kaftandjian, J. Rinkel, An optimised method for material identification using a photon counting detector, Nuclear Instruments and Methods in Physics Research, Section A: Accelerators, Spectrometers, Detectors and Associated Equipment 663 (2012) 26-36.

[26] X. Wang, J. Xu, E. C. Frey, Optimization of energy window widths in basis material decomposition using a multi-window photon counting X-ray detector, in: 2007 IEEE Nuclear Science Symposium Conference Record, volume 5, IEEE, 2007, pp. 3826-3829. doi:10.1109/ NSSMIC. 2007.4436955.

[27] C. P. A. Brambilla, A. Gorecki, A. Potop, L. Verger, Basis material decomposition method for material discrimination with a new spectrometric X-ray imaging detector, Journal of Instrumentation 12 (2017) P08014.

[28] M. Busi, K. A. Mohan, A. A. Dooraghi, K. M. Champley, H. E. Martz, U. L. Olsen, Method for system-independent material characterization from spectral X-ray CT, NDT \& E International 107 (2019) 102136.

[29] A. S. Wang, N. J. Pelc, Optimal energy thresholds and weights for separating materials using photon counting x-ray detectors with energy discriminating capabilities, in: Medical Imaging 2009: Physics of Medical Imaging, volume 7258, International Society for Optics and Photonics, 2009, p. 725821. doi:10.1117/12.811454.

[30] A. S. Wang, N. J. Pelc, Sufficient statistics as a generalization of binning in spectral X-ray imaging, IEEE Transactions on Medical Imaging 30 (2011) 84-93.

[31] R. F. Eilbert, K. D. Krug, Dual energy x-ray scanner for detecting contraband, in: Contraband and Cargo Inspection Technology International Symposium Proceedings, 1992, pp. 209-217.

[32] Busi, Matteo, Enhanced Security Screening Using Spectral X-ray Imaging, Phd thesis, Technical University of Denmark, 2019.

[33] M. Kheirabadi, A. Bjorholm Dahl, U. Lund Olsen, W. Mustafa, M. Lyksborg, Multispectral X-ray CT: multivariate statistical analysis for efficient reconstruction, in: Developments in X-Ray Tomography XI, volume 1039113, International Society for Optics and Photonics, 2017, p. 38. doi:10.1117/12.2273338.

[34] S. Bragg, WH and Peirce, LXIV. The absorption coefficients of X rays, The London, Edinburgh, and Dublin Philosophical Magazine and Journal of Science 28 (1914) 626-630.

[35] E. Owen, The absorption of x-rays, Proceedings of the Royal Society of London. Series A, Containing Papers of a Mathematical and Physical Character 94 (1918) 510-524.

[36] F. K. Richtmyer, F. W. Warburton, The absorption of X-rays by iron, cobalt, nickel and copper, Physical Review 22 (1923) 539-545.

[37] D. White, An analysis of the Z-dependence of photon and electron interactions, Physics in Medicine $\backslash \&$ Biology 22 (1977) 219.

[38] A. H. Compton, S. K. Allison, et al., X-rays in theory and experiment, New York, NY, USA: Van Nostrand (1935).

[39] Mayneord, WV, The significance of the roentgen, Acta of the International Union Against Cancer 2 (1937) 271.

[40] F. W. SPIERS, Effective atomic number and energy absorption in tissues., The British journal of radiology 19 (1946) 52-63.

[41] E. C. McCullough, Photon attenuation in computed tomography, Medical Physics 2 (1975) 307-320.

[42] J. Weber, D. J. van den Berge, The effective atomic number and the calculation of the composition of phantom materials., The British journal of radiology 42 (1969) 378-383. 
[43] Z. H. Cho, C. M. Tsai, G. Wilson, Study of contrast and modulation mechanisms in X-ray/photon transverse axial transmission tomography, Physics in Medicine and Biology 20 (1975) 879-889.

[44] A. Lehmann, LA and Alvarez, RE and Macovski, Aetal and Brody, WR and Pelc, NJ and Riederer, Stephen J and Hall, Generalized image combinations in dual KVP digital radiography, Medical physics 8 (1981) 659-667.

[45] J. F. Williamson, S. Li, S. Devic, B. R. Whiting, F. A. Lerma, On two-parameter models of photon cross sections: Application to dualenergy CT imaging, Medical Physics 33 (2006) 4115-4129.

[46] D. J. Walter, E. J. Tkaczyk, X. Wu, Accuracy and precision of dual energy CT imaging for the quantification of tissue fat content, in: Medical Imaging 2006: Physics of Medical Imaging, volume 6142, International Society for Optics and Photonics, 2006, p. 61421G. doi: $10.1117 / 12.654669$.

[47] W. G. Langeveld, Effective Atomic Number, Mass Attenuation Coefficient Parameterization, and Implications for High-Energy X-Ray Cargo Inspection Systems, Physics Procedia 90 (2017) 291-304.

[48] E. S. Dreier, J. Kehres, M. Khalil, M. Busi, Y. Gu, R. Feidenhans, U. L. Olsen, Spectral correction algorithm for multispectral CdTe X-ray detectors, Optical Engineering 57 (2018) 16.

[49] M. M. Goodsitt, E. G. Christodoulou, S. C. Larson, Accuracies of the synthesized monochromatic CT numbers and effective atomic numbers obtained with a rapid $\mathrm{kVp}$ switching dual energy CT scanner, Medical Physics 38 (2011) 2222-2232.

[50] S. Kim, J. Chen, T. Cheng, A. Gindulyte, J. He, S. He, Q. Li, B. A. Shoemaker, P. A. Thiessen, B. Yu, L. Zaslavsky, J. Zhang, E. E. Bolton, PubChem 2019 update: Improved access to chemical data, 2019. URL: https://pubchem. ncbi.nlm. nih. gov. doi:10.1093/ nar/gky1033.

[51] W. J. Palenstijn, K. J. Batenburg, J. Sijbers, The ASTRA tomography toolbox, in: 13th International Conference on Computational and Mathematical Methods in Science and Engineering, CMMSE, volume 2013, 2013, pp. 1139-1145. URL: http://www.visielab.ua.ac. be/sites/default/files/cmmse2013.pdf.

[52] K. Berger, M.J., Hubbell, J.H., Seltzer, S.M., Chang, J., Coursey, J.S., Sukumar, R., Zucker, D.S., and Olsen, XCOM: Photon Cross Section Database, 2010. URL: http://physics.nist.gov/xcom. doi:10.18434/ T4866X.

[53] J. Nocedal, S. J. Wright, Numerical Optimization, Springer, New York, 1999.

[54] R. A. Rutherford, B. R. Pullan, I. Isherwood, Measurement of effective atomic number and electron density using an EMI scanner, Neuroradiology 11 (1976) 15-21.

[55] M. E. Phelps, M. H. Gado, E. J. Hoffman, Correlation of effective atomic number and electron density with attenuation coefficients measured with polychromatic X rays, Radiology 117 (1975) 585-588.

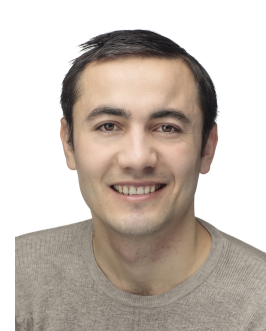

Doniyor is a PhD fellow at the Department of Physics at Denmark Technical University (DTU), Kongens Lyngby, Denmark. He obtained his BS degree in physics from the National University of Uzbekistan, Tashkent, Uzbekistan, and his MS degree in Molecular Photonics for bio- and nanotechnologies (MONABIPHOT) from the École Normale Supérieure de Cachan, Cachan, France, in 2012 and 2018, respectively.

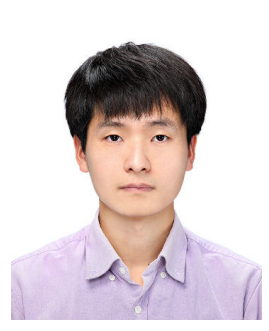

Jakeoung Koo received his B.Sc. and M.Sc. in Computer Science from Chung-Ang University, South Korea in 2015 and 2017, respectively. He is now a Ph.D. student in Technical University of Denmark (DTU), Kongens Lyngby, Denmark. He is interested in Computed Tomography and Computer Vision.

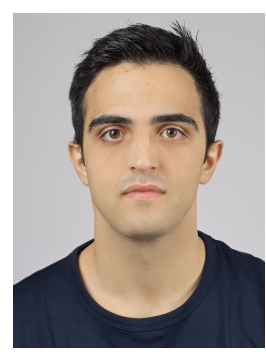

Matteo Busi is a postdoctoral researcher at the Paul Scherrer Institute (Switzerland) since 2019. He was awarded his $\mathrm{PhD}$ degree in 2019 from the Technical University of Denmark, for his contributions towards the development and enhancement of spectral X-ray imaging techniques. He is currently researching on the development of novel neutron Bragg edge imaging techniques and respective applications in the material science domain, especially in the field of additive manufacturing.



Professor, Dr. techn. Henning Friis Poulsen is employed at DTU Physics since 2012. Henning pioneered several $\mathrm{x}$-ray microscopy methods enabling mapping of materials properties within large samples, and on scales down to nanometres. He heads the 3D Imaging Centre at DTU and is Principal Investigator on a number of European and national projects with the aim of introducing X-ray methods to industry.

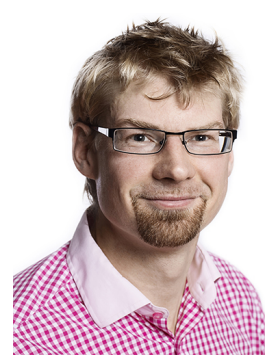

Ulrik is a senior research engineer at DTU's department of Physics since 2013. He obtained his Ph.D. degree in 2009 from Copenhagen University. $\mathrm{He}$ is an expert in X-ray instrumentation and specialises in spectral $\mathrm{X}$-ray detectors, data acquisition and processing. Ulrik's current research interest is the use of spectral detectors for security applications for improved reconstruction and material identification.

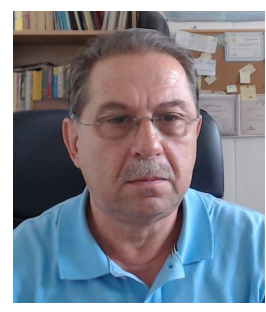

Mihai IOVEA, Engineer in Physics, Senior Research Scientist, Ph.D in Physics Over 30 years' experience in X-Ray Imaging projects research $\&$ development for Security and Non-Destructive Testing. Interested in X-ray 2D\&3D Tomography and Digital Radiography techniques, such as: dual/multi energy, diffraction, back-scattering applied in for threats detection, high-resolution in-line X-ray scanners for fast industrial nondestructive testing. 